\title{
Some Results on p-Shape Curvatures of Non-Lightlike Space Curves
}

\author{
Hakan Şimşek* and Mustafa Özdemir \\ (Communicated by Kazım İlarslan)
}

\begin{abstract}
In this paper, we obtain some findings for a non-null curve parameterized by spherical arc length. We investigate the relationship between a non-null curve with p-shape curvatures and pseudospherical curves on $S_{1}^{2}$ and $H^{2}(-1)$. We introduce the concept of similar helix in Minkowski 3-space $\mathbb{E}_{1}^{3}$. Besides, we explicitly determine the parametrizations of all non-lightlike self-similar curves by using the pseudo-spherical curves in $\mathbb{E}_{1}^{3}$.
\end{abstract}

Keywords: -shape curvature; -similarity; helix.

AMS Subject Classification (2010): Primary: 53A35 ; Secondary: 53A55; 53B30.

\section{Introduction}

A similarity transformation (or similitude) of the Euclidean space is an automorphism preserving the angles and ratios between lengths. The geometric properties unchanged by similarity transformations is called the similarity geometry. The Euclidean geometry is actually a special case of similarity geometry. Similarity transformations are used for curve matching, which is an important research area in the computer vision and pattern recognition. Also, the recognition and pose determination of $3 \mathrm{D}$ objects can be represented by space curves are required for industry automation, robotics, navigation and medical applications (see $[6,22,1,15,16])$. On the other hand, a self-similar object, which is exactly similar to a part of itself, recently have been a considerable issue in some areas such as fractal geometry, dynamical systems, computer networks (see $[18,9,10,4])$.

The broad content of similarity transformations were given by [5] in the Euclidean space. The differential geometric invariants of space curves according to the group of similarities were studied by [7] in the Euclidean 3 -space. Also, the similarity geometry of Frenet curves was extended to the Eucliedan and Minkowski $n$-spaces by [8] and [23], respectively. On the other hand, the studies [13] and [14] investigated the concept of helix (or cylindrical helix) and slant helix in Euclidean 3-space. The paper [2] represented the Lorentzian version of slant helix in Minkowski 3-space.

The content of paper is as follows. We give some information regarding the similarity geometry of non-null curves in $\mathbb{E}_{1}^{3}$. In the next section, we examine a relation between a non-lightlike space curve $\alpha$ and its pseudospherical tangential indicatrix $\mathbf{c}$ and we find that the $\mathrm{p}$-shape torsion of $\alpha$ is equal to the geodesic curvature of c. Furthermore, we show that the pseudo-spherical Darboux images of $\alpha$ coincide with the pseudo-spherical evolutes of $\mathbf{c}$. We represent the notion of similar helix and give a characterization of the similar helix in $\mathbb{E}_{1}^{3}$. Lastly, using the tangent indicatrix, we obtain the parametrizations of all non-lightlike self-similar curves, whose $\mathrm{p}$-shapes are real constants.

\section{Preliminaries}

Firstly, let us give some basic notions of the Lorentzian geometry. Let $\mathbf{x}=\left(x_{1}, x_{2}, x_{3}\right)$ and $\mathbf{y}=\left(y_{1}, y_{2}, y_{3}\right)$ be arbitrary two vectors in $\mathbb{E}_{1}^{3}$. The Lorentzian inner product of $\mathbf{x}$ and $\mathbf{y}$ can be stated as $\mathbf{x} \cdot \mathbf{y}=\mathbf{x} I^{*} \mathbf{y}^{T}$ where 
$I^{*}=\operatorname{diag}(-1,1,1)$. The vector $\mathbf{x}$ in $\mathbb{E}_{1}^{3}$ is called a spacelike, lightlike (or null) and timelike vector if $\mathbf{x} \cdot \mathbf{x}>0$ or $\mathbf{x}=0, \mathbf{x} \cdot \mathbf{x}=0$ or $\mathbf{x} \cdot \mathbf{x}<0$, respectively. The norm of the vector $\mathbf{x}$ is given by $\|\mathbf{x}\|=\sqrt{|\mathbf{x} \cdot \mathbf{x}|}$. The Lorentzian vector product $\mathbf{x} \times \mathbf{y}$ of $\mathbf{x}$ and $\mathbf{y}$ is defined as

$$
\mathbf{x} \times \mathbf{y}=\left[\begin{array}{ccc}
-i & j & k \\
x_{1} & x_{2} & x_{3} \\
y_{1} & y_{2} & y_{3}
\end{array}\right] .
$$

We can describe the pseudo-spheres in $\mathbb{E}_{1}^{3}$ as follows: The hyperbolic 2-space is defined by

$$
H^{2}(-1)=\left\{\mathbf{x} \in \mathbb{E}_{1}^{3}: \mathbf{x} \cdot \mathbf{x}=-1\right\}
$$

and de Sitter 2-space is defined by

$$
S_{1}^{2}=\left\{\mathbf{x} \in \mathbb{E}_{1}^{3}: \mathbf{x} \cdot \mathbf{x}=1\right\} .
$$

The hyperbolic 2-space has two components,

$$
H_{+}^{2}(-1)=\left\{\mathbf{x} \in H^{2}(-1): x_{1} \geq 1\right\} \text { and } H_{-}^{2}(-1)=\left\{\mathbf{x} \in H^{2}(-1): x_{1} \leq 1\right\}
$$

(see [19]).

A pseudo-similarity (p-similarity) of $\mathbb{E}_{1}^{3}$ is a composition of a homothety (dilatation), a pseudo-orthogonal map and a translation. Then, any p-similarity $f: \mathbb{E}_{1}^{3} \rightarrow \mathbb{E}_{1}^{3}$ can be expressed by

$$
f(x)=\mu \mathcal{A} x+\mathbf{b}
$$

where $\mathcal{A}$ is a pseudo-orthogonal matrix with $\operatorname{det} \mathcal{A}=1, \mu>0$ is a scaling factor and $\mathbf{b}$ is a translation (see [23]). Then, we have $\|\vec{f}(\mathbf{u})\|=|\mu|\|\mathbf{u}\|$ for any $\mathbf{u} \in \mathbb{E}_{1}^{3}$ where $\vec{f}(\overrightarrow{x y})=\overrightarrow{f(x) f(y)}$ (see [5]). The constant $|\mu|$ is called a $p$-similarity ratio of $f$. The p-similarities form a group under the composition of maps and denoted by $\operatorname{Sim}\left(\mathbb{E}_{1}^{3}\right)$. The group of orientation-preserving (reversing) p-similarities is denoted by $\operatorname{Sim}^{+}\left(\mathbb{E}_{1}^{3}\right)\left(\operatorname{Sim}^{-}\left(\mathbb{E}_{1}^{3}\right)\right.$, resp.). The p-similarity transformations preserve the causal characters and angles. On the other hand, the p-similarities can also be represented by using split quaternion algebra. Let $\hat{H}$ be the split quaternion algebra and $\mathbb{T H}$ be the set of timelike split quaternions such that we identify $\mathbb{E}_{1}^{3}$ with $\operatorname{Im} \hat{\mathbb{H}}$. $\mathbb{T} \hat{H}$ is a group under the split quaternion product. There exists a unit timelike split quaternion $q$ by [20] such that the transformation $\mathbf{R}_{q}: \operatorname{Im} \mathbb{T} \hat{H} \rightarrow \operatorname{Im} T \hat{H}$ defined by

$$
\mathbf{R}_{q}(r)=q r q^{-1}
$$

can be regarded as a rotation in the Minkowski 3-space. Thus, a p-similarity has the representation

$$
f(r)=\mu q r q^{-1}+\mathbf{b}
$$

for some fixed $\mu \neq 0 \in \mathbb{R}$ and $\mathbf{b} \in \operatorname{Im} \hat{\mathbb{H}} \cong \mathbb{E}_{1}^{3}$.

Let $\alpha: t \in J \rightarrow \alpha(t) \in \mathbb{E}_{1}^{3}$ be a non-lightlike space curve of class $C^{3}$ and $\kappa$ and $\tau$ show its curvature and torsion, respectively. The Frenet-Serret formulas of $\alpha$ in $\mathbb{E}_{1}^{3}$ according to the arc-length parameter $s$ are given by

$$
\frac{d}{d s}\left[\begin{array}{l}
\mathbf{e}_{1} \\
\mathbf{e}_{2} \\
\mathbf{e}_{3}
\end{array}\right]=\left[\begin{array}{ccc}
0 & \kappa & 0 \\
\varepsilon_{\mathbf{e}_{3} \kappa} & 0 & \tau \\
0 & \varepsilon_{\mathbf{e}_{1}} \tau & 0
\end{array}\right]\left[\begin{array}{l}
\mathbf{e}_{1} \\
\mathbf{e}_{2} \\
\mathbf{e}_{3}
\end{array}\right]
$$

where $\left\{\mathbf{e}_{1}, \mathbf{e}_{2}, \mathbf{e}_{3}\right\}$ is a Frenet frame of $\alpha$ and $\varepsilon_{\mathbf{e}_{\ell}}=\mathbf{e}_{\ell} \cdot \mathbf{e}_{\ell}$ for $1 \leq \ell \leq 3$ (see [11] and [21]). The Darboux vector of the non-lightlike curve is expressed by $D(s)=\varepsilon_{\mathbf{e}_{3}} \tau(s) \mathbf{e}_{1}(s)+\varepsilon_{\mathbf{e}_{2}} \kappa(s) \mathbf{e}_{3}(s)$. We suppose that $D(s)$ is a nonlightlike vector. The normalization of Darboux vector given by

$$
\frac{D}{\|D\|}=\frac{\varepsilon_{\mathbf{e}_{3}} \tau \mathbf{e}_{1}+\varepsilon_{\mathbf{e}_{2}} \kappa \mathbf{e}_{3}}{\sqrt{\left|\varepsilon_{\mathbf{e}_{1}} \tau^{2}+\varepsilon_{\mathbf{e}_{3}} \kappa\right|}}
$$

is called pseudo-spherical Darboux image of $\alpha$ or Lorentzian Darboux indicatrix of $\alpha$. In this section, the differentiation according to $s$ is denoted by primes. The curvature $\kappa$ and torsion $\tau$ of $\alpha$ are given by

$$
\kappa(s)=\left\|\alpha^{\prime \prime}\right\|, \quad \tau(s)=\frac{\operatorname{det}\left(\alpha^{\prime}, \alpha^{\prime \prime}, \alpha^{\prime \prime \prime}\right)}{\left\|\alpha^{\prime} \times \alpha^{\prime \prime}\right\|^{2}} .
$$


Definition 2.1. ([17]) A helix $\alpha: J \rightarrow \mathbb{E}_{1}^{3}$ is a regular curve parameterized by arc-length such that there exists a vector $\mathbf{v} \in \mathbb{E}_{1}^{3}$ with the property that the function $\left\langle\mathbf{e}_{1}, \mathbf{v}\right\rangle$ is constant.

Theorem 2.1. ([17]) Let $\alpha: J \rightarrow \mathbb{E}_{1}^{3}$ be a Frenet curve. Then $\alpha$ is a helix if and only if $\tau / \kappa$ is constant.

If we denote the image of $\alpha$ under a p-similarity $f \in \operatorname{Sim}\left(\mathbb{E}_{1}^{3}\right)$ by $\beta$, then $\beta$ can be given as

$$
\beta(t)=\mu q \alpha(t) q^{-1}+\mathbf{b}, \quad t \in J .
$$

The arc length functions of $\alpha$ and $\beta$ starting at $t_{0} \in J$ are

$$
s(t)=\int_{t_{0}}^{t}\left\|\frac{d \alpha(u)}{d u}\right\| d u, \quad s^{*}(t)=\int_{t_{0}}^{t}\left\|\frac{d \beta(u)}{d u}\right\| d u=|\mu| s(t) .
$$

Any non-lightlike curve with non-zero curvature can be reparameterized by an arc-length parameter of its tangent indicatrix called the spherical arc-length parameter of $\alpha$.

Lemma 2.1. ([23]) Let $\sigma$ be the spherical arc-length parameter of $\alpha$. The spherical arc-length element $d \sigma$ and the functions $\tilde{\kappa}=-\frac{d \kappa}{\kappa d \sigma}$ and $\tilde{\tau}=\frac{\tau}{\kappa}$ are invariants under the group of p-similarities of the Minkowski 3-space.

Definition 2.2. Let $\alpha: I \rightarrow \mathbb{E}_{1}^{3}$ be a non-lightlike space curve of the class $C^{3}$ parameterized by the spherical arc length parameter $\sigma$. Let $\kappa(\sigma)$ and $\tau(\sigma)$ be the curvature and torsion of $\alpha$, respectively. The functions

$$
\tilde{\kappa}=-\frac{d \kappa}{\kappa d \sigma} \quad \text { and } \quad \tilde{\tau}=\frac{\tau}{\kappa}
$$

are called $p$-shape curvature and $p$-shape torsion of $\alpha$. Also, the ordered pair $(\tilde{\kappa}, \tilde{\tau})$ is called a (local) $p$-shape of the non-lightlike curve $\alpha$ in the Minkowski 3-space.

The Lemma 2.1 implies that we can use the spherical arc length parameter and the p-shape of $\alpha$ in order to study the geometry of a non-lightlike curve under the p-similarity map. So, the derivative formulas of the curve $\alpha$ with respect to $\sigma$ are given by

$$
\frac{d \alpha}{d \sigma}=\frac{1}{\kappa} \mathbf{e}_{1}, \quad \frac{d^{2} \alpha}{d \sigma^{2}}=\tilde{\kappa} \frac{d \alpha}{d \sigma}+\frac{1}{\kappa} \mathbf{e}_{2}
$$

and

$$
\frac{d}{d \sigma}\left[\begin{array}{l}
\mathbf{e}_{1} \\
\mathbf{e}_{2} \\
\mathbf{e}_{3}
\end{array}\right]=\left[\begin{array}{ccc}
0 & 1 & 0 \\
\varepsilon_{\mathbf{e}_{3}} & 0 & \tilde{\tau} \\
0 & \varepsilon_{\mathbf{e}_{1}} \tilde{\tau} & 0
\end{array}\right]\left[\begin{array}{l}
\mathbf{e}_{1} \\
\mathbf{e}_{2} \\
\mathbf{e}_{3}
\end{array}\right]
$$

The formulas (2.8) and (2.9) are also valid for non-lightlike curve $\beta$. Additionally, the similarity invariants $\tilde{\kappa}$ and $\tilde{\tau}$ can also be founded as

and

$$
\tilde{\kappa}(\sigma)=\frac{\frac{d^{2} \alpha}{d \sigma^{2}} \cdot \frac{d \alpha}{d \sigma}}{\frac{d \alpha}{d \sigma} \cdot \frac{d \alpha}{d \sigma}}
$$

respectively.

Two non-lightlike space curves which have the same torsion and the same positive curvature are always equivalent according to a Lorentzian motion. This notion can be extended for the non-lightlike space curves which have the same $p$-shape curvature and $p$-shape torsion under a Lorentzian similarity motion in $\mathbb{E}_{1}^{3}$. We have the following existence and uniqueness theorems proved in [23].

Theorem 2.2. Let $\alpha, \alpha^{*}: I \rightarrow \mathbb{E}_{1}^{3}$ be two non-lightlike space curves of class $C^{3}$ parameterized by the same spherical arc length parameter $\sigma$ and have the same casual characters, where $I \subset \mathbb{R}$ is an open interval. Suppose that $\alpha$ and $\alpha^{*}$ have the same $p$-shape curvatures $\tilde{\kappa}=\tilde{\kappa}^{*}$ and the same $p$-shape torsions $\tilde{\tau}=\tilde{\tau}^{*}$ for any $\sigma \in$ I. If $\alpha$ and $\alpha^{*}$ are timelike or spacelike curves, then there exists a p-similarity $f \in \operatorname{Sim}^{-}\left(\mathbb{E}_{1}^{3}\right)$ or $f \in \operatorname{Sim}^{+}\left(\mathbb{E}_{1}^{3}\right)$ such that $\alpha^{*}=f \circ \alpha$. 
Theorem 2.3. Let $z_{i}: I \rightarrow \mathbb{R}, i=1,2$, be two functions of class $C^{1}$ and $\mathbf{e}_{1}^{0}, \mathbf{e}_{2}^{0}, \mathbf{e}_{3}^{0}$ be an right-handed orthonormal triad of vectors at a point $x_{0}$ in $\mathbb{E}_{1}^{3}$. According to a p-similarity, there exists a unique non-lightlike curve $\alpha: I \rightarrow \mathbb{E}_{1}^{3}$ such that $\alpha$ satisfies the following conditions:

(i) There exists $\sigma_{0} \in I$ such that $\alpha\left(\sigma_{0}\right)=x_{0}$ and the Frenet-Serret frame of $\alpha$ at $x_{0}$ is $\left\{\mathbf{e}_{1}^{0}, \mathbf{e}_{2}^{0}, \mathbf{e}_{3}^{0}\right\}$.

(ii) $\tilde{\kappa}(\sigma)=z_{1}(\sigma)$ and $\tilde{\tau}(\sigma)=z_{2}(\sigma)$ for any $\sigma \in I$.

Remark 2.1. We consider the pseudo-orthogonal 3-frame $\left\{\mathbf{e}_{1} / \kappa, \mathbf{e}_{2} / \kappa, \mathbf{e}_{3} / \kappa\right\}$, for the non-lightlike curve parameterized by $\sigma$. Then, by the equations (2.8) and (2.9), we get

$$
\frac{d}{d \sigma_{\alpha}}\left[\begin{array}{l}
\mathbf{e}_{1} / \kappa \\
\mathbf{e}_{2} / \kappa \\
\mathbf{e}_{3} / \kappa
\end{array}\right]=\left[\begin{array}{ccc}
\tilde{\kappa} & 1 & 0 \\
\varepsilon_{\mathbf{e}_{3}} & \tilde{\kappa} & \tilde{\tau} \\
0 & \varepsilon_{\mathbf{e}_{1}} \tilde{\tau} & \tilde{\kappa}
\end{array}\right]\left[\begin{array}{l}
\mathbf{e}_{1} / \kappa \\
\mathbf{e}_{2} / \kappa \\
\mathbf{e}_{3} / \kappa
\end{array}\right] .
$$

The pseudo-orthogonal frame $\left\{\mathbf{e}_{1} / \kappa, \mathbf{e}_{2} / \kappa, \mathbf{e}_{3} / \kappa\right\}$ is invariant under the group $\operatorname{Sim}\left(\mathbb{E}_{1}^{3}\right)$. Thus, we can consider the equation (2.12) as the Frenet-Serret frame of $\alpha$ under the Lorentzian similarity motion.

\section{The Construction of Similar Curves}

Let c : $I \rightarrow S_{1}^{2}$ be a non-lightlike pseudo-spherical curve with the arc length parameter $\sigma$. The orthonormal frame $\{\mathbf{c}(\sigma), \mathbf{t}(\sigma), \mathbf{q}(\sigma)\}$ along $\mathbf{c}$ is called the Lorentzian Sabban frame of $\mathbf{c}$ if $\mathbf{t}(\sigma)=\frac{d \mathbf{c}}{d \sigma}$ is the unit tangent vector of $\mathbf{c}$ and $\mathbf{q}(\sigma)=\mathbf{c}(\sigma) \times \mathbf{t}(\sigma)$. Then, we can state the pseudo-spherical Frenet-Serret formulas of the non-lightlike curve $\mathbf{c}$.

If the curve $\mathbf{c}$ is a timelike curve, i.e. $\mathbf{t}(\sigma)$ is timelike vector, then we have the following pseudo-spherical Frenet-Serret formulas of $\mathbf{c}$ :

$$
\frac{d}{d \sigma}\left[\begin{array}{l}
\mathbf{c} \\
\mathbf{t} \\
\mathbf{q}
\end{array}\right]=\left[\begin{array}{ccc}
0 & 1 & 0 \\
1 & 0 & \kappa_{g} \\
0 & \kappa_{g} & 0
\end{array}\right]\left[\begin{array}{l}
\mathbf{c} \\
\mathbf{t} \\
\mathbf{q}
\end{array}\right] .
$$

If $\mathbf{q}(\sigma)$ is a timelike vector, then the pseudo-spherical Frenet-Serret formulas of $\mathbf{c}$ are given by

$$
\frac{d}{d \sigma}\left[\begin{array}{l}
\mathbf{c} \\
\mathbf{t} \\
\mathbf{q}
\end{array}\right]=\left[\begin{array}{ccc}
0 & 1 & 0 \\
-1 & 0 & -\kappa_{g} \\
0 & -\kappa_{g} & 0
\end{array}\right]\left[\begin{array}{l}
\mathbf{c} \\
\mathbf{t} \\
\mathbf{q}
\end{array}\right] .
$$

If $\mathbf{c}: I \rightarrow H_{+}^{2}(-1)$ is a spacelike curve with the arc length parameter $\sigma$, then pseudo-spherical Frenet-Serret formulas of $\mathbf{c}$ are given by

$$
\frac{d}{d \sigma}\left[\begin{array}{l}
\mathbf{c} \\
\mathbf{t} \\
\mathbf{q}
\end{array}\right]=\left[\begin{array}{ccc}
0 & 1 & 0 \\
1 & 0 & \kappa_{g} \\
0 & -\kappa_{g} & 0
\end{array}\right]\left[\begin{array}{l}
\mathbf{c} \\
\mathbf{t} \\
\mathbf{q}
\end{array}\right]
$$

where $\mathbf{c}(\sigma)$ is a timelike vector. $\kappa_{g}(\sigma)=\operatorname{det}\left(\mathbf{c}(\sigma), \mathbf{t}(\sigma), \frac{d \mathbf{t}}{d \sigma}(\sigma)\right)$ is the geodesic curvature of $\mathbf{c}$ for three different Frenet-Serret formulas.

Let $k: I \rightarrow \mathbb{R}$ be a function of class $C^{1}$. We can construct a non-lightlike curve $\alpha: I \rightarrow \mathbb{E}_{1}^{3}$ defined by

$$
\alpha(\sigma)=b \int e^{\int k(\sigma) d \sigma} \mathbf{c}(\sigma) d \sigma+\mathbf{a},
$$

where $\mathbf{a}$ is a constant vector and $b$ is a real constant. It can be easily seen that $\sigma$ is the spherical arc length parameter of $\alpha$.

Proposition 3.1. The non-lightlike curve $\alpha$ defined by (3.1) is a Frenet curve whose $p$-shape curvature and p-shape torsion are given by $\tilde{\kappa}=k(\sigma)$ and $\tilde{\tau}=\kappa_{g}(\sigma)$ in the Minkowski 3-space, respectively. Furthermore, all non-lightlike Frenet curves can be obtained in this way. 
Proof. First, from (3.1) we can write

$$
\begin{aligned}
\frac{d \alpha}{d \sigma} & =b e^{\int k(\sigma) d \sigma} \mathbf{c}(\sigma) \\
\frac{d^{2} \alpha}{d \sigma^{2}} & =b e^{\int k(\sigma) d \sigma}\left[k(\sigma) \mathbf{c}(\sigma)+\frac{d \mathbf{c}}{d \sigma}\right] \text { and } \\
\frac{d^{3} \alpha}{d \sigma^{3}} & =b e^{\int k(\sigma) d \sigma}\left[\left\{k^{2}(\sigma)+\frac{d k}{d \sigma}\right\} \mathbf{c}(\sigma)+2 k(\sigma) \frac{d \mathbf{c}}{d \sigma}+\frac{d^{2} \mathbf{c}}{d \sigma^{2}}\right] .
\end{aligned}
$$

Then, because of the equation

$$
\frac{d \alpha}{d \sigma} \times \frac{d^{2} \alpha}{d \sigma^{2}}=b^{2} e^{2 \int k(\sigma) d \sigma}\left(\mathbf{c}(\sigma) \times \frac{d \mathbf{c}}{d \sigma}\right) \neq 0,
$$

we say that $\alpha$ is a non-lightlike Frenet curve. Using (2.10), (2.11) and (3.2), we find

$$
\tilde{\kappa}=k(\sigma) \text { and } \tilde{\tau}=\operatorname{det}\left(\mathbf{c}, \frac{d \mathbf{c}}{d \sigma}, \frac{d \mathbf{t}}{d \sigma}\right)=\kappa_{g}(\sigma) .
$$

Conversely, suppose that $\alpha: I \rightarrow \mathbb{E}_{1}^{3}$ is a non-lightlike Frenet curve parameterized by a spherical arc length parameter $\sigma$. Denote the curvature and the torsion of $\alpha$ by $\kappa(\sigma)$ and $\tau(\sigma)$, respectively. Let $\mathbf{c}$ be the spherical indicatrix of $\alpha$ such that $\mathbf{c}: I \rightarrow \mathbb{E}_{1}^{3}$ is given by

$$
\mathbf{c}(\sigma)=\mathbf{e}_{1}(\sigma)=\frac{\frac{d \alpha}{d \sigma}}{\left\|\frac{d \alpha}{d \sigma}\right\|}=\kappa(\sigma) \frac{d \alpha}{d \sigma} .
$$

Then, $\sigma$ is an arc length parameter of $\mathbf{c}$ and using (2.9) and (3.4) we get

$$
\kappa_{g}=\operatorname{det}\left(\mathbf{c}(\sigma), \mathbf{t}(\sigma), \frac{d \mathbf{t}(\sigma)}{d \sigma}\right)=\operatorname{det}\left(\mathbf{e}_{1}(\sigma), \mathbf{e}_{2}(\sigma), \varepsilon_{\mathbf{e}_{3}} \mathbf{e}_{1}(\sigma)+\tilde{\tau} \mathbf{e}_{3}\right)=\tilde{\tau},
$$

which is the geodesic curvature of $\mathbf{c}$. If we take $k(\sigma)=\tilde{\kappa}(\sigma)$, then

$$
\begin{aligned}
\int e^{\int k(\sigma) d \sigma} \mathbf{c}(\sigma) d \sigma & =\int e^{\int-\frac{d \kappa}{\kappa d \sigma} d \sigma} \mathbf{c}(\sigma) d \sigma=e^{b_{0}} \int \frac{1}{\kappa} \mathbf{c}(\sigma) d \sigma \\
& =e^{b_{0}} \int \frac{d \alpha}{d \sigma} d \sigma=e^{b_{0}} \alpha(\sigma)+\mathbf{a}_{0}
\end{aligned}
$$

where $b_{0}$ is a real constant and $\mathbf{a}_{0}$ is a constant vector. Hence, we can write

$$
\alpha(\sigma)=b \int e^{\int k(\sigma) d \sigma} \mathbf{c}(\sigma) d \sigma+\mathbf{a} \text {. }
$$

Corollary 3.1. The non-lightlike pseudo spherical curve $\mathbf{c}$ is a pseudo-circle if and only if the corresponding non-lightlike curve defined by (3.1) is a helix.

Proof. It is obvious from the equation $\kappa_{g}(\sigma)=\tilde{\tau}(\sigma)=\frac{\tau(\sigma)}{\kappa(\sigma)}=$ const.

Now, we shall give another relation between the pseudo-spherical curve c and the curve defined by (3.1). For this reason, we consider the pseudo-spherical evolutes of c. The hyperbolic evolute of $\mathbf{c}: I \rightarrow H_{+}^{2}(-1)$ is defined by

$$
H \mathcal{E}_{\mathbf{c}}(\sigma)=\frac{1}{\sqrt{\left|\kappa_{g}^{2}(\sigma)-1\right|}}\left(\kappa_{g}(\sigma) \mathbf{c}(\sigma)+\mathbf{q}(\sigma)\right)
$$

under the assumption that $\kappa_{g}(\sigma) \neq \pm 1$. Note that $H \mathcal{E}_{\mathbf{c}}(\sigma)$ is located in $H_{+}^{2}(-1) \cup H_{-}^{2}(-1)$ if and only if $\kappa_{g}^{2}(\sigma)>1$. If $H \mathcal{E}_{\mathbf{c}}(\sigma)$ is located in $H_{-}^{2}(-1)$, then it may be considered $-H \mathcal{E}_{\mathbf{c}}(\sigma)$ instead of $H \mathcal{E}_{\mathbf{c}}(\sigma)$ (see [12]). Besides, the de Sitter evolute of a timelike curve c : $I \rightarrow S_{1}^{2}$ is defined by

$$
D \mathcal{E}_{\mathbf{c}}(\sigma)=\frac{1}{\sqrt{\kappa_{g}^{2}(\sigma)+1}}\left(\kappa_{g}(\sigma) \mathbf{c}(\sigma)-\mathbf{q}(\sigma)\right)
$$

on $S_{1}^{2}$ (see [3]). 
Proposition 3.2. i) Let $\mathbf{c}: I \rightarrow H_{+}^{2}(-1)$ be a pseudo-spherical spacelike curve and a non-lightlike curve $\alpha: I \rightarrow \mathbb{E}_{1}^{3}$ be a corresponding curve defined by (3.1). Then, the pseudo-spherical Darboux image of $\alpha$ coincides with the hyperbolic evolute of $\mathbf{c}$.

ii) Let $\mathbf{c}: I \rightarrow S_{1}^{2}$ be a pseudo-spherical timelike curve on the de Sitter 2-space and a non-lightlike curve $\alpha: I \rightarrow \mathbb{E}_{1}^{3}$ be a corresponding curve defined by (3.1). Then, the pseudo-spherical Darboux image of $\alpha$ coincides with the de Sitter evolute of $\mathbf{c}$.

Proof. The corresponding curve $\alpha$ is a timelike curve because of $\mathbf{e}_{1}(\sigma)=\mathbf{c}(\sigma)$. Using the equation (2.3), the pseudo-spherical Darboux image of $\alpha$ is

$$
D_{0}=\frac{1}{\sqrt{\left|1-\tilde{\tau}^{2}\right|}}\left(\tilde{\tau} \mathbf{e}_{1}+\mathbf{e}_{3}\right) .
$$

We observe that $\mathbf{e}_{3}(\sigma)=\mathbf{c}(\sigma) \times \frac{d \mathbf{c}}{d \sigma}=\mathbf{q}(\sigma)$ by the equation (3.3). Thus, we can obtain

$$
D_{0}(\sigma)=\frac{1}{\sqrt{\left|\kappa_{g}^{2}(\sigma)-1\right|}}\left(\kappa_{g}(\sigma) \mathbf{c}(\sigma)+\mathbf{q}(\sigma)\right)=H \mathcal{E}_{\mathbf{c}}(\sigma) .
$$

ii) Since we know

$$
D_{0}=\frac{1}{\sqrt{\left|\tilde{\tau}^{2}+1\right|}}\left(\tilde{\tau} \mathbf{e}_{1}-\mathbf{e}_{3}\right)
$$

from the equation $(2.3)$, it can be seen the equality $D_{0}=D \mathcal{E}_{\mathbf{c}}(\sigma)$.

Now, we define a notion of similar helix for a non-lightlike curve parameterized by spherical arc length in the Minkowski 3-space.

Definition 3.1. A similar helix (S-helix) $\alpha: I \rightarrow \mathbb{E}_{1}^{3}$ is a regular curve parameterized by spherical arc length such that there exists a vector $\mathbf{v} \in \mathbb{E}_{1}^{3}$ with the property that the function $\left\langle\frac{1}{\kappa} \mathbf{e}_{1}, \mathbf{v}\right\rangle$ is constant, where $\kappa$ is the curvature of $\alpha$.

Theorem 3.1. Let $\alpha: I \rightarrow \mathbb{E}_{1}^{3}$ be a non-lightlike Frenet curve parameterized by a spherical arc length $\sigma$. Then $\alpha$ is a S-helix if and only if the function

$$
\left(\frac{\kappa-\varepsilon_{\mathbf{e}_{3}}\left(d^{2} \kappa / d \sigma^{2}\right)}{\tilde{\tau}}\right)^{2}-\left(\varepsilon_{\mathbf{e}_{1}}+\frac{\varepsilon_{\mathbf{e}_{2}}}{\tilde{\kappa}^{2}}\right)\left(\frac{d \kappa}{d \sigma}\right)^{2}
$$

is constant.

Proof. We first assume that the curve $\alpha$ is a S-helix. Let $\mathbf{v} \in \mathbb{E}_{1}^{3}$ be a vector satisfying the equation $\left\langle\frac{1}{\kappa} \mathbf{e}_{1}, \mathbf{v}\right\rangle=c$, where $c$ is a non-zero real constant. Then, there exist smooth functions $q_{2}$ and $q_{3}$ such that

$$
\mathbf{v}=\left(c \kappa^{2}(\sigma)\right) \frac{1}{\kappa(\sigma)} \mathbf{e}_{1}(\sigma)+q_{2}(\sigma) \frac{1}{\kappa(\sigma)} \mathbf{e}_{2}(\sigma)+q_{3}(\sigma) \frac{1}{\kappa(\sigma)} \mathbf{e}_{3}(\sigma), \sigma \in I .
$$

By differentiating the vector $\mathbf{v}$ together $(2.12)$ and using the constancy of $\mathbf{v}$, we get

$$
\begin{aligned}
& 2 c \kappa d \kappa / d \sigma+c \kappa^{2} \tilde{\kappa}+\varepsilon_{\mathbf{e}_{3}} q_{2}=0, \\
& c \kappa^{2}+d q_{2} / d \sigma+q_{2} \tilde{\kappa}+\varepsilon_{\mathbf{e}_{1}} q_{3} \tilde{\tau}=0,=0 . \\
& q_{2} \tilde{\tau}+d q_{3} / d \sigma+q_{3} \tilde{\kappa}=0 .
\end{aligned}
$$

Also, we have

$$
\langle\mathbf{v}, \mathbf{v}\rangle=\varepsilon_{\mathbf{e}_{1}} c^{2}+\frac{1}{\kappa^{2}}\left(\varepsilon_{\mathbf{e}_{2}} q_{2}^{2}+\varepsilon_{\mathbf{e}_{3}} q_{3}^{2}\right)=m=\text { constant. }
$$

The first equation of (3.8) gives $q_{2}=-\varepsilon_{\mathbf{e}_{3}} c \kappa d \kappa / d \sigma$ and therefore we get

$$
q_{3}= \pm \sqrt{\varepsilon_{\mathbf{e}_{1}} c^{2} \kappa^{2}\left(\frac{d \kappa}{d \sigma}\right)^{2}+\varepsilon_{\mathbf{e}_{2}} c^{2} \kappa^{4}+\varepsilon_{\mathbf{e}_{3}} m \kappa^{2}}
$$


by using the equation (3.9). Then, we can find the following identity by putting $q_{2}$ and $q_{3}$ into the second equation of $(3.8)$

$$
c \kappa^{2}-\varepsilon_{\mathbf{e}_{3}} c \kappa \frac{d^{2} \kappa}{d \sigma^{2}}=\mp \varepsilon_{\mathbf{e}_{1}} \tilde{\tau} \sqrt{\varepsilon_{\mathbf{e}_{1}} c^{2} \kappa^{2}\left(\frac{d \kappa}{d \sigma}\right)^{2}+\varepsilon_{\mathbf{e}_{2}} c^{2} \kappa^{4}+\varepsilon_{\mathbf{e}_{3}} m \kappa^{2}}
$$

and after some computation we conclude

$$
\left(\frac{\kappa-\varepsilon_{\mathbf{e}_{3}}\left(d^{2} \kappa / d \sigma^{2}\right)}{\tilde{\tau}}\right)^{2}-\left(\varepsilon_{\mathbf{e}_{1}}+\frac{\varepsilon_{\mathbf{e}_{2}}}{\tilde{\kappa}^{2}}\right)\left(\frac{d \kappa}{d \sigma}\right)^{2}=\frac{\varepsilon_{\mathbf{e}_{3}} m}{c^{2}},
$$

which means that the function (3.7) is constant .

Conversely, let's assume the opposite case. If we define a vector

$$
\mathbf{v}=c \kappa^{2}\left(\frac{1}{\kappa} \mathbf{e}_{1}\right)-\varepsilon_{\mathbf{e}_{3}} c \kappa \frac{d \kappa}{d \sigma}\left(\frac{1}{\kappa} \mathbf{e}_{2}\right) \pm \sqrt{\varepsilon_{\mathbf{e}_{1}} c^{2} \kappa^{2}\left(\frac{d \kappa}{d \sigma}\right)^{2}+\varepsilon_{\mathbf{e}_{2}} c^{2} \kappa^{4}+\varepsilon_{\mathbf{e}_{3}} \kappa^{2}}\left(\frac{1}{\kappa} \mathbf{e}_{3}\right),
$$

we get $d \mathbf{v} / d \sigma=0$, that is, $\mathbf{v}$ is a constant vector. On the other hand, $\alpha$ is a S-helix as we have $\left\langle\frac{1}{\kappa(\sigma)} \mathbf{e}_{1}(\sigma), \mathbf{v}\right\rangle=$ $c=$ constant.

\subsection{Forming of a Non-lightlike Curve by Its $p$-shape}

Let $\alpha: I \rightarrow \mathbb{E}_{1}^{3}$ be a non-lightlike curve with the spherical arc length parameter $\sigma$ such that the ordered pair $(\tilde{\kappa}, \tilde{\tau})$ is p-shape of $\alpha$ defined by (2.7). First, we define a fixed orthonormal triad of the non-lightlike vectors $\mathbf{e}_{1}^{0}, \mathbf{e}_{2}^{0}, \mathbf{e}_{3}^{0}$. We take one of the following differential equations depending on whether $\mathbf{t}(\sigma), \mathbf{c}(\sigma)$ or $\mathbf{q}(\sigma)$ is a timelike vector, respectively

$$
\begin{aligned}
& \frac{d \mathbf{c}}{d \sigma}=\mathbf{t}(\sigma), \quad \frac{d \mathbf{t}}{d \sigma}=\mathbf{c}(\sigma)+\tilde{\tau} \mathbf{q}(\sigma), \quad \frac{d \mathbf{q}}{d \sigma}=\tilde{\tau} \mathbf{t}(\sigma) \\
& \frac{d \mathbf{c}}{d \sigma}=\mathbf{t}(\sigma), \quad \frac{d \mathbf{t}}{d \sigma}=\mathbf{c}(\sigma)+\tilde{\tau} \mathbf{q}(\sigma), \quad \frac{d \mathbf{q}}{d \sigma}=-\tilde{\tau} \mathbf{t}(\sigma) \\
& \frac{d \mathbf{c}}{d \sigma}=\mathbf{t}(\sigma), \quad \frac{d \mathbf{t}}{d \sigma}=-\mathbf{c}(\sigma)-\tilde{\tau} \mathbf{q}(\sigma), \quad \frac{d \mathbf{q}}{d \sigma}=-\tilde{\tau} \mathbf{t}(\sigma) .
\end{aligned}
$$

The unique solution of one of these differential equations with initial conditions $\mathbf{e}_{1}^{0}, \mathbf{e}_{2}^{0}, \mathbf{e}_{3}^{0}$ determine a spherical non-lightlike curve $\mathbf{c}=\mathbf{c}(\sigma)$ such that $\mathbf{c}\left(\sigma_{0}\right)=\mathbf{e}_{1}^{0}$ for some $\sigma_{0} \in I$. Let $\rho(\sigma)=\int_{\sigma_{1}}^{\sigma} \tilde{\kappa}(\sigma) d \sigma$ for fixed $\sigma_{1} \in I$. Using the equation (3.1) and proposition 3.1, we can find the non-lightlike curve

$$
\alpha(\sigma)=\alpha_{0}+\int_{\sigma_{0}}^{\sigma} e^{\rho(\sigma)} \mathbf{c}(\sigma) d \sigma
$$

passes through a point $\alpha_{0}=\alpha\left(\sigma_{0}\right)$. Let's see an example of the non-lightlike curve constructed by above procedure.

Example 3.1. Let $\alpha: I \rightarrow \mathbb{E}_{1}^{3}$ be a non-lightlike curve with p-shape $(\tilde{\kappa}, \tilde{\tau})=(1 / \sigma, a)$ where $a \neq 0$ is real constant. We take the unit vector $\mathbf{t}(\sigma)$ as a timelike vector and choose initial conditions

$$
\mathbf{e}_{1}^{0}=\left(0,-\frac{1}{\sqrt{1+a^{2}}}, \frac{a}{\sqrt{1+a^{2}}}\right), \mathbf{e}_{2}^{0}=(1,0,0), \mathbf{e}_{3}^{0}=\left(0, \frac{a}{\sqrt{1+a^{2}}}, \frac{1}{\sqrt{1+a^{2}}}\right) .
$$

Then, the system (3.10) describes a spherical timelike curve c : $I \rightarrow S_{1}^{2}$ defined by

$$
\mathbf{c}(\sigma)=\left(\frac{1}{\sqrt{1+a^{2}}} \sinh \left(\sqrt{1+a^{2}} \sigma\right),-\frac{1}{\sqrt{1+a^{2}}} \cosh \left(\sqrt{1+a^{2}} \sigma\right), \frac{a}{\sqrt{1+a^{2}}}\right)
$$

Because of $\rho(\sigma)=\ln \sigma$, the parametric equation of the non-lightlike curve $\alpha$ is given by

$$
\alpha(\sigma)=\left(\frac{u \cosh u-\sinh u}{\left(1+a^{2}\right)^{3 / 2}}, \frac{\cosh u-u \sinh u}{\left(1+a^{2}\right)^{3 / 2}}, \frac{a u^{2}}{2\left(1+a^{2}\right)^{3 / 2}}\right)
$$

where $u=\sqrt{1+a^{2}} \sigma$. 
3.1.1. Classification of Non-lightlike Self-Similar Curves A non-lightlike curve $\alpha: I \rightarrow \mathbb{E}_{1}^{3}$ is called self-similar if any p-similarity $f \in G$ conserve globally $\alpha$ and $G$ acts transitively on $\alpha$ where $G$ is a one-parameter subgroup of $\operatorname{Sim}\left(\mathbb{E}_{1}^{3}\right)$. This means that p-shape curvatures are constant. In fact, let $p_{1}=\alpha\left(s_{1}\right)$ and $p_{2}=\alpha\left(s_{2}\right)$ be two different points lying on $\alpha$. Since $G$ acts transitively on $\alpha$, there is a similarity $f \in G$ such that $f\left(p_{1}\right)=p_{2}$. Then, we find $\tilde{\kappa}\left(s_{1}\right)=\tilde{\kappa}\left(s_{2}\right)$ and $\tilde{\tau}\left(s_{1}\right)=\tilde{\tau}\left(s_{2}\right)$.

Case 1: Let p-shape $(\tilde{\kappa}, \tilde{\tau})$ of the $\alpha: I \rightarrow \mathbb{E}_{1}^{3}$ be $(0,0)$. The equation (3.13) determine the pseudo-circles which are the hyperbolas on the timelike plane and the circles on the spacelike plane.

Case 2: We let $(\tilde{\kappa}, \tilde{\tau})=(0, a)$ where $a \neq 0$ is real constant.

i) We take the unit vector $\mathbf{t}(\sigma)$ as a timelike vector with the initial conditions (3.14). Solving the equation (3.13), we obtain the spacelike self-similar curve parameterized by

$$
\alpha_{1}(\sigma)=\left(\frac{1}{q^{2}} \cosh (q \sigma),-\frac{1}{q^{2}} \sinh (q \sigma), \frac{a}{q} \sigma\right), \quad \sigma \in I
$$

where $q=\sqrt{1+a^{2}}$.

ii) Let the unit vector $\mathbf{c}(\sigma)$ be a timelike vector and $a^{2}>1$. We choose the initial conditions

$$
\mathbf{e}_{1}^{0}=\left(\frac{a}{\sqrt{a^{2}-1}}, 0, \frac{1}{\sqrt{a^{2}-1}}\right), \mathbf{e}_{2}^{0}=(0,1,0), \mathbf{e}_{3}^{0}=\left(\frac{1}{\sqrt{a^{2}-1}}, 0, \frac{a}{\sqrt{a^{2}-1}}\right) .
$$

Then, the system (3.11) describes a pseudo-spherical spacelike curve c : $I \rightarrow H_{0}^{2}$ defined by

$$
\mathbf{c}(\sigma)=\left(\frac{a}{\sqrt{a^{2}-1}}, \frac{1}{\sqrt{a^{2}-1}} \sin \left(\sqrt{a^{2}-1} \sigma\right), \frac{1}{\sqrt{a^{2}-1}} \cos \left(\sqrt{a^{2}-1} \sigma\right)\right)
$$

with $\mathbf{c}(0)=\mathbf{e}_{1}^{0}$, in the Minkowski 3-space. From the equation (3.13), the timelike self-similar curve is given by

$$
\alpha_{2}(\sigma)=\left(\frac{a}{n_{1}} \sigma,-\frac{1}{n_{1}^{2}} \cos \left(n_{1} \sigma\right), \frac{1}{n_{1}^{2}} \sin \left(n_{1} \sigma\right)\right)
$$

where $n_{1}=\sqrt{a^{2}-1}$.

If we have $a^{2}<1$, the pseudo-spherical spacelike curve $\mathbf{c}: I \rightarrow H_{0}^{2}$ can be found as

$$
\mathbf{c}(\sigma)=\left(\frac{1}{\sqrt{1-a^{2}}} \cosh \left(\sqrt{1-a^{2}} \sigma\right), \frac{1}{\sqrt{1-a^{2}}} \sinh \left(\sqrt{1-a^{2}} \sigma\right), \frac{a}{\sqrt{1-a^{2}}}\right)
$$

with the initial conditions

$$
\mathbf{e}_{1}^{0}=\left(\frac{1}{\sqrt{1-a^{2}}}, 0, \frac{a}{\sqrt{1-a^{2}}}\right), \mathbf{e}_{2}^{0}=(0,1,0), \mathbf{e}_{3}^{0}=\left(\frac{a}{\sqrt{1-a^{2}}}, 0, \frac{1}{\sqrt{1-a^{2}}}\right)
$$

so that $\mathbf{c}(0)=\mathbf{e}_{1}^{0}$. So, we get a timelike self-similar curve given by

$$
\alpha_{3}(\sigma)=\left(\frac{1}{n_{2}^{2}} \sinh \left(n_{2} \sigma\right), \frac{1}{n_{2}^{2}} \cosh \left(n_{2} \sigma\right), \frac{a}{n_{2}} \sigma\right)
$$

where $n_{2}=\sqrt{1-a^{2}}$.

iii) Let the unit vector $\mathbf{q}(\sigma)$ be a timelike vector and $a^{2}>1$. Choosing the initial conditions

$$
\mathbf{e}_{1}^{0}=\left(\frac{1}{n_{1}}, 0, \frac{a}{n_{1}}\right), \mathbf{e}_{2}^{0}=(0,1,0), \mathbf{e}_{3}^{0}=\left(\frac{a}{n_{1}}, 0, \frac{1}{n_{1}}\right),
$$

we obtain a spacelike self-similar curve given by

$$
\alpha_{4}(\sigma)=\left(\frac{1}{n_{1}^{2}} \sinh \left(n_{1} \sigma\right), \frac{1}{n_{1}^{2}} \cosh \left(n_{1} \sigma\right), \frac{a}{n_{1}} \sigma\right) .
$$

Analogously, if $a^{2}<1$, then we get a timelike self-similar curve

$$
\alpha_{5}(\sigma)=\left(\frac{a}{n_{2}} \sigma, \frac{-1}{n_{2}^{2}} \cos \left(n_{2} \sigma\right), \frac{1}{n_{2}^{2}} \sin \left(n_{2} \sigma\right)\right) .
$$


Case 3: Let $\alpha: I \rightarrow \mathbb{E}_{1}^{3}$ be a non-lightlike curve with the p-shape $(\tilde{\kappa}, \tilde{\tau})=(b, a)$ where $b \neq 0$ and $a$ are real constants.

i) we take $\mathbf{t}(\sigma)$ as a timelike unit vector. Choosing initial conditions (3.14), we get the same spherical timelike curve (3.15) which is a pseudo-circle with a radius $1 / \sqrt{1+a^{2}}$. Solving the equation (3.13), we obtain a spacelike self-similar curve as the following

$$
\alpha_{\mathbf{t}}(\sigma)=\left(\frac{e^{b \sigma}}{\left(b^{2}-q^{2}\right)}\left(\frac{b}{q} \sinh (q \sigma)-\cosh (q \sigma)\right), \frac{e^{b \sigma}}{\left(b^{2}-q^{2}\right)}\left(\sinh (q \sigma)-\frac{b}{q} \cosh (q \sigma)\right), \frac{a}{b q} e^{b \sigma}\right) .
$$

ii) If we take $\mathbf{c}(\sigma)$ as a timelike unit vector and $a^{2}>1$, by using (3.16), we get a timelike self-similar curve given by

$$
\alpha_{\mathbf{c}_{1}}(\sigma)=\left(\frac{a}{b n_{1}} e^{b \sigma}, \frac{e^{b \sigma}}{\left(b^{2}-n_{1}^{2}\right)}\left(\frac{b}{n_{1}} \sin \left(n_{1} \sigma\right)-\cos \left(n_{1} \sigma\right)\right), \frac{e^{b \sigma}}{\left(b^{2}-n_{1}^{2}\right)}\left(\frac{b}{n_{1}} \cos \left(n_{1} \sigma\right)+\sin \left(n_{1} \sigma\right)\right)\right)
$$

If $a^{2}<1$, by using (3.17), a timelike self-similar curve can be obtained as

$$
\alpha_{\mathbf{c}_{2}}(\sigma)=\left(\frac{e^{b \sigma}}{\left(b^{2}-n_{2}^{2}\right)}\left(\frac{b}{n_{2}} \cosh \left(n_{2} \sigma\right)-\sinh \left(n_{2} \sigma\right)\right), \frac{e^{b \sigma}}{\left(b^{2}-n_{2}^{2}\right)}\left(\frac{b}{n_{2}} \sinh \left(n_{2} \sigma\right)-\cosh \left(n_{2} \sigma\right)\right), \frac{a}{b n_{2}} e^{b \sigma}\right) .
$$

iii) If we take $\mathbf{q}(\sigma)$ as a timelike unit vector and $a^{2}>1$, we get a spacelike self-similar curve

$$
\alpha_{\mathbf{q}_{1}}(\sigma)=\left(\frac{e^{b \sigma}}{\left(b^{2}-n_{1}^{2}\right)}\left(\frac{b}{n_{1}} \cosh \left(n_{1} \sigma\right)-\sinh \left(n_{1} \sigma\right)\right), \frac{e^{b \sigma}}{\left(b^{2}-n_{1}^{2}\right)}\left(\frac{b}{n_{1}} \sinh \left(n_{1} \sigma\right)-\cosh \left(n_{1} \sigma\right)\right), \frac{a}{b n_{1}} e^{b \sigma}\right) .
$$

If $a^{2}<1$, by using (3.17), a spacelike self-similar curve can be obtained as

$$
\alpha_{\mathbf{q}_{2}}(\sigma)=\left(\frac{a}{b n_{2}} e^{b \sigma}, \frac{e^{b \sigma}}{\left(b^{2}-n_{2}^{2}\right)}\left(\frac{b}{n_{2}} \sin \left(n_{2} \sigma\right)-\cos \left(n_{2} \sigma\right)\right), \frac{e^{b \sigma}}{\left(b^{2}-n_{2}^{2}\right)}\left(\frac{b}{n_{2}} \cos \left(n_{2} \sigma\right)+\sin \left(n_{2} \sigma\right)\right)\right) .
$$

It is obvious that the lines are self-similar curves. Thus, we conclude that the non-lightlike self-similar curves in the Minkowski 3-space are the lines, the pseudo-circles and the curves parameterized by $\alpha_{i}$ for $i=1, \cdots, 5$, $\alpha_{\mathbf{t}}, \alpha_{\mathbf{c}_{1}}, \alpha_{\mathbf{c}_{2}}, \alpha_{\mathbf{q}_{1}}$ and $\alpha_{\mathbf{q}_{2}}$. So, we have the following result.

Theorem 3.2. Let $\alpha$ be a non-lightlike curve in $\mathbb{E}_{1}^{3}$. Then, $\alpha$ is a self-similar curve if and only if it is a line or pseudo-circle or similar to one of the curves $\alpha_{\mathbf{t}}, \alpha_{\mathbf{c}_{1}}, \alpha_{\mathbf{c}_{2}}, \alpha_{\mathbf{q}_{1}}, \alpha_{\mathbf{q}_{2}}$ and $\alpha_{i}$ for $i=1, \cdots, 5$.

\section{References}

[1] Alcázar, J. G., Hermosoa, C. and Muntingh, G., Detecting similarity of rational plane curves, Journal of Computational and Applied Mathematics, 269 (2014), 1-13.

[2] Ali, A. T. and Lopez, R., Slant Helices in Minkowski Space $\mathbb{E}_{1}^{3}$, J. Korean Math. Soc. 48 (2011), 159-167.

[3] Babaarslan, M. and Yayl1, Y., Time-Like Constant Slope Surfaces and Space-Like Bertrand Curves in Minkowski 3-Space, Proc. Natl. Acad. Sci., India, Sect. A Phys. Sci., 84 (2014), 535-540.

[4] Barnsley, M. F., Hutchinson, J. E. and Stenflo, Ö., V-variable fractals: Fractals with partial self similarity, Advances in Mathematics, 218 (2008), 2051-2088.

[5] Berger, M., Geometry I. Springer, New York, 1998.

[6] Brook, A., Bruckstein, A. M. and Kimmel, R., On Similarity-Invariant Fairness Measures, LNCS, 3459 (2005), $456-467$.

[7] Encheva, R. and Georgiev, G., Shapes of space curves, J. Geom. Graph., 7 (2003), 145-155.

[8] Encheva, R. and Georgiev, G., Similar Frenet curves, Results in Mathematics, 55 (2009), 359-372.

[9] Hutchinson, J. E., Fractals and Self-Similarity, Indiana University Mathematics Journal, 30 (1981), N:5.

[10] K. Falconer, K., Fractal Geometry: Mathematical Foundations and Applications, Second Edition, John Wiley \& Sons, Ltd., 2003.

[11] Inoguchi, J., Biharmonic curves in Minkowski 3-space, International Journal of Mathematics and Mathematical Sciences, 21 (2003), $1365-1368$.

[12] Izumiya, S., Pei, D., Sano, T. and Torii E., Evolutes of Hyperbolic Plane Curves, Acta Mathematica Sinica, English Series, 20 (2004), 543-550.

[13] Izumiya, S. and Takeuchi, N., Generic properties of helices and Bertrand curves, J. Geom., 74 (2002), 97-109.

[14] Izumiya, S. and Takeuchi, N., New special curves and developable surfaces, Turkish J. Math., 28 (2004), 153-163.

[15] Li, S. Z., Invariant Representation, Matching and Pose Estimation of 3D Space Curves Under Similarity Transformation, Pattern Recognition, 30 (1997), 447-458.

[16] Li, S. Z., Similarity Invariants for 3D Space Curve Matching, In Proceedings of the First Asian Conference on Computer Vision, Japan (1993), 454-457. 
[17] López, R., Differential Geometry of Curves and Surfaces in Lorentz-Minkowski Space, International Electronic Journal of Geometry, 7 (2014), 44-107.

[18] Mandelbrot, B. B., The Fractal Geometry of Nature, New York: W. H. Freeman, 1983.

[19] O'Neill, B., Semi-Riemannian Geometry with Applications to Relativity, Academic Press Inc., London, 1983.

[20] Özdemir, M., Ergin, A. A., Rotations with unit timelike quaternions in Minkowski 3-space, Journal of Geometry and Physics, 56 (2006), 322-336.

[21] Özdemir, M., Ergin, A. A., Spacelike Darboux Curves in Minkowski 3-Space, Differ. Geom. Dyn. Syst., 9 (2007), $131-137$.

[22] Sahbi, H., Kernel PCA for similarity invariant shape recognition, Neurocomputing, 70 (2007), $3034-3045$.

[23] Şimşek, H. and Özdemir, M., Similar and Self-Similar Curves in Minkowski n-Space, B. Korean Math. Soc., 52 (2015), $2071-2093$.

\section{Affiliations}

HAKAN ŞIMSSEK

ADDRESS: Antalya Bilim University, Dept. of Industrial Engineering, Antalya-TURKEY

E-MAIL: hakan.simsek@antalya.edu.tr

\section{ORCID ID : orcid.org/0000-0002-1028-2676}

Mustafa ÖZDEMIR

ADDRESS: Akdeniz University, Dept. of Mathematics, Antalya-TURKEY

E-MAIL: mozdemir@akdeniz.edu.tr

ORCID ID : orcid.org/0000-0002-1359-4181 